\title{
Nanoscale tin dioxide films and zinc oxide hierarchical nanostructures for gas sensing applications
}

\author{
N.P. Klochko, K.S. Klepikova, G.S. Khrypunov, O.V. Pirohov, V.A. Novikov \\ National Technical University (NTU "KhPI") \\ 21, Frunze str., 61002 Kharkiv, Ukraine
}

\begin{abstract}
Nanoscale tin dioxide $\left(\mathrm{SnO}_{2}\right)$ and zinc oxide $(\mathrm{ZnO})$ layers are considered as promising candidates for preparation of sensing elements for metal oxide semiconductor gas sensors. Tin dioxide films deposited by direct current magnetron sputtering are investigated. The influence of deposition temperature and annealing on the structure and electrical properties of the tin dioxide films are considered. The development of design and technological solution of active layer with high gas sensitivity, reproducibility and stability is offered. Studies of effects of the pulse electrodeposition regimes on structural and substructural parameters and on morphology of zinc oxide arrays made it possible to identify modes that are optimal for formation of hierarchical nanostructures with large specific surface area suitable for gas sensing applications.
\end{abstract}

Keywords: tin dioxide, crystal structure, annealing, active layer, zinc oxide, hierarchical nanostructure.

Manuscript received 12.03.14; revised version received 15.08.14; accepted for publication 29.10.14; published online 10.11.14.

\section{Introduction}

Nanoscale tin dioxide $\left(\mathrm{SnO}_{2}\right)$ and zinc oxide $(\mathrm{ZnO})$ layers are promising candidates for preparation of sensing elements for metal oxide semiconductor gas sensors [1-6]. Semiconducting metal oxide thin films exhibit changes in their electrical conductivity with small amount of reducing or oxidizing gases, which provide sensors with several advantages such as low cost, short response time, wide range of target gases and high sensitivity $[1,7]$.

As compared with other metal oxides, tin dioxide and zinc oxide exhibit gas sensitive properties at relatively low temperatures $\left(100 \ldots 350{ }^{\circ} \mathrm{C}\right)[2,4]$, which provides a low power consumption of the sensors. Chemical durability of tin dioxide allows using the $\mathrm{SnO}_{2}$ based gas sensors for detection of the majority of controlled gaseous impurities in the air including the aggressive ones. The abovementioned properties provide $\mathrm{SnO}_{2}$ based gas sensors with a long lifetime [1-5]. Preparation of tin dioxide thin films by a magnetron sputtering is one of the most promising vacuum technologies for microelectronics [8-11]. This method fits well with other technological operations of modern micro- and nanoelectronics. However, a competition of the tin dioxide magnetron sputtering with $\mathrm{SnO}_{2}$ thickfilm technology for the gas sensor creation and with production of the gas sensing elements based on sintered $\mathrm{SnO}_{2}$ powders is not so successful in the production scale $[2,5-6]$. A limited understanding in the literature of the mechanisms for a control of the magnetron sputtered $\mathrm{SnO}_{2}$ layers structure characteristics that determine their gas sensitivity hinder solution of problems encountered in their commercialization.

Another promising material for the active layer of the gas sensor is zinc oxide due to its tendency toward formation of different one-dimensional (1D) nanostructures, such as nanowires, nanorods, nanoribbons, nanotubes etc., because it is known that semiconducting materials with high surface-to-volume ratios are particularly well suited for resistive type gas sensors. One of the most promising ways of increasing the specific area of $1 \mathrm{D} \mathrm{ZnO}$ arrays is to produce multiscale hierarchical nanostructures on the base of $1 \mathrm{D}$ 
$\mathrm{ZnO}$. The hierarchical nanostructures of $\mathrm{ZnO}$ have a large surface area-to-volume ratio, which is an advantage for maximizing the surface response. The hierarchical approach consists of the combination of one-dimensional $\mathrm{ZnO}$ nanostructures with even smaller $\mathrm{ZnO}$ nanoparticles or nanoporous $\mathrm{ZnO}$ layers [12, 13]. With the aim to grow zinc oxide hierarchical nanostructures, the increasing interest is being drawn not only to expensive methods such as vacuum condensation [13], but also to high efficient and inexpensive electrochemical methods [12]. This is because the growth of zinc oxide nanostructures by using this method combines such advantages as simple processing equipment, applicability to mass production and process controllability that are very important for fabrication of gas sensors. So, one purpose of this work was to investigate the magnetron sputtering parameters effect on the crystal structure of gas sensitive tin dioxide films and to study their electrical properties during heat treatment. The second aim was to determine the effects of the pulse electrolysis modes on the morphology and structural parameters of the zinc oxide layers to implement the controlled growth of hierarchical zinc oxide nanostructures with large specific surface area in the pulsed plating modes.

\section{Experimental}

Tin dioxide thin films were obtained using direct current magnetron sputtering of a target prepared as a pressed $\mathrm{SnO}_{2}$ powder in $\mathrm{Ar}-\mathrm{O}_{2}$ gas atmosphere at the 2-Pa residual pressure. Three sets of film samples (S1, S2, S3) with the thickness $(h)$ close to $450 \mathrm{~nm}$ at the substrate temperatures $\left(T_{\text {sub }}\right): 150,250$ and $350{ }^{\circ} \mathrm{C}$, respectively, were prepared. Also, the series $\mathrm{S} 4$ film samples were made with the thickness $h 50 \mathrm{~nm}$, which were deposited at $T_{\text {sub }}=150{ }^{\circ} \mathrm{C}$. The structure and phase composition of the films were investigated by $\mathrm{X}$-ray diffraction analysis (XRD) using "DRON-4M" with a monochromatic $\mathrm{CuK}_{\alpha}$ radiation. Structural studies of the films were also carried out with a transmission electron microscope EMV-100L in accord with the standard technique. A conventional graphite replica method was used for researching the structure of $\mathrm{SnO}_{2}$ thin films magnetron sputtered on pyroceramic and alumina ceramic substrates. Since the tin dioxide films have a very high adhesion to these substrates, their separation from the substrates and direct investigation of the film structure in a transmission electron microscope was not possible. Therefore, for their studies we have used "witnesses" on $\mathrm{NaCl}$ and mica substrates. The $\mathrm{SnO}_{2}$ films sputtered on the $\mathrm{NaCl}$ substrates were easily obtained by the salt dissolving in the water. The mica substrates were cleaved to a thickness at which $\mathrm{SnO}_{2} /$ mica becomes transparent to electrons.

Surface morphology of the obtained layers was investigated with a scanning electron microscope "REM$100 \mathrm{U}$ " in the secondary and backscattered electrons registration modes at $30 \mathrm{kV}$ accelerating voltage. Since the samples were mainly of high resistivity, so the surface of the $\mathrm{SnO}_{2}$ samples was coated by vacuum deposited conductive layers to prevent accumulation of the electric charge, namely, by $\mathrm{Au}, \mathrm{Al}$ and graphite served as coating materials that should not mask the minute details of the $\mathrm{SnO}_{2}$ microstructure. The thickness of the conductive $\mathrm{Au}, \mathrm{Al}$ or graphite layer was specially chosen for achieving the layer with electrical continuity. The electric conductivity measurements of tin dioxide films were carried out using a digital voltmeter and digital storage oscilloscope.

Zinc oxide arrays were obtained by cathodic electrochemical deposition in the three-electrode electrochemical cell with an unmixed aqueous electrolyte containing $0.05 \mathrm{M} \quad \mathrm{Zn}\left(\mathrm{NO}_{3}\right)_{2}$ and $0.1 \mathrm{M}$ $\mathrm{NaNO}_{3}$ at temperatures within the range $30 \ldots 85^{\circ} \mathrm{C}$. Glass plates coated with transparent conductive layers of fluorine doped tin oxide (hereafter, FTO) (Pilkington Corp., USA) were used as substrates. A platinum spiral was used as a counter electrode; a saturated silver chloride electrode $\mathrm{Ag} / \mathrm{AgCl}$ was used as a reference electrode. Samples were prepared by the pulse electrolysis method by applying rectangular potential pulses to the system cathode-substrate, so that the lower and upper potential limits were $U_{\text {off }}=-0.8 \mathrm{~V}$ and $U_{\text {on }}=$ $-1.4 \mathrm{~V}$. Upon the electrodeposition of each individual sample for $1 \mathrm{~h}$, the pulse frequency $f$ was kept constant within the limits of $2 \mathrm{~Hz}$ to $1 \mathrm{kHz}$.

To analyze $\mathrm{ZnO}$ layer structure, $\mathrm{X}$-ray spectra were obtained using a "DRON-4M" diffractometer in $\mathrm{CoK}_{\alpha}$ radiation with the Bragg-Brentano focusing. The sizes of grains as coherent scattering regions (CSRs) and microstrains $\Delta d / d$ (where $d$ is the crystal lattice parameter according to JCPCDS; $\Delta d$ is the difference between the experimental and reference values for the crystal lattice parameter) in the zinc oxide arrays were estimated using the Williamson-Hall approximation. The texture of the electrodeposited zinc oxide arrays was studied through pole density $P_{i}$ by defining the number of crystallites in which the $(h k l)$ planes are parallel to the sample surface. The surface morphology of the zinc oxide layers was studied by intermittent-contact atomic force microscopy (AFM) using a "NanoLaboratory NTEGRA Prima NT-MDT” setup.

\section{Results and discussion}

\subsection{Tin dioxide films}

\subsubsection{Effect of the substrate temperature and humidity of the atomizing gases on the $\mathrm{SnO}_{2}$ structure}

Our studies have shown that magnetron sputtered tin dioxide layers deposited at $50 \%$ oxygen partial pressure in argon atmosphere onto relatively little heated substrates $\left(T_{\text {sub }}=100{ }^{\circ} \mathrm{C}\right)$ are amorphous immediately after their magnetron sputtering. This is evidenced by both absorption contrast in the electron microscopic picture and by the presence of a halo on the electron diffraction pattern (Fig. 1a). The amorphous layers do 
not have atomically smooth surface and consist of flakes with the average size $15 \mathrm{~nm}$. A distinctive feature of these structures is the lack of intimate contact between them, no regular geometric shapes and no sharp outlines. Such $\mathrm{SnO}_{2}$ films represent conglomerates consisting of smaller particles (amorphous globules) with dimensions of about 1 to $2 \mathrm{~nm}$. This feature of their shape is highlighted in the schematic Fig. 1b. It can be assumed that observed flake formation is an example of the coagulation accretion of the particles [5], when the mechanical association into a conglomerate of the amorphous globules that contact with each other occurs without their complete transformation into a spherical particle. In this case, the films are metastable not only due to its amorphous structure but also because of their relatively high specific surface area. In this case, the change of the surface-to-volume ratio during the transition from the insular condensation step to continuous layer formation occurs only due to appearance of common joints between grains. So, the decrease in the surface free energy is negligible. There are plenty of cavities (pores) inside the conglomerates, which can contain adsorbed residual gases. A cause of the loosely arranged conglomerates, apparently, is a low vacuum (near $2 \mathrm{~Pa}$ ) required for the magnetron sputtering. Therefore, the atomizing gas adsorbed on the surface probably prevents coalescence.

Tin dioxide layers deposited at higher substrate temperature $\left(T_{s u b}=300{ }^{\circ} \mathrm{C}\right)$ consist of flat-shaped particles (Fig. 1c). This is evidenced by the homogeneous blackening within each particle. These particles have curved contours with more regular shape in comparison with amorphous flakes obtained on the little heated substrates. Particle sizes $(15 \mathrm{~nm})$ roughly correspond to the dimensions of the previously described conglomerates represented in Figs $1 \mathrm{a}$ and $1 \mathrm{~b}$.
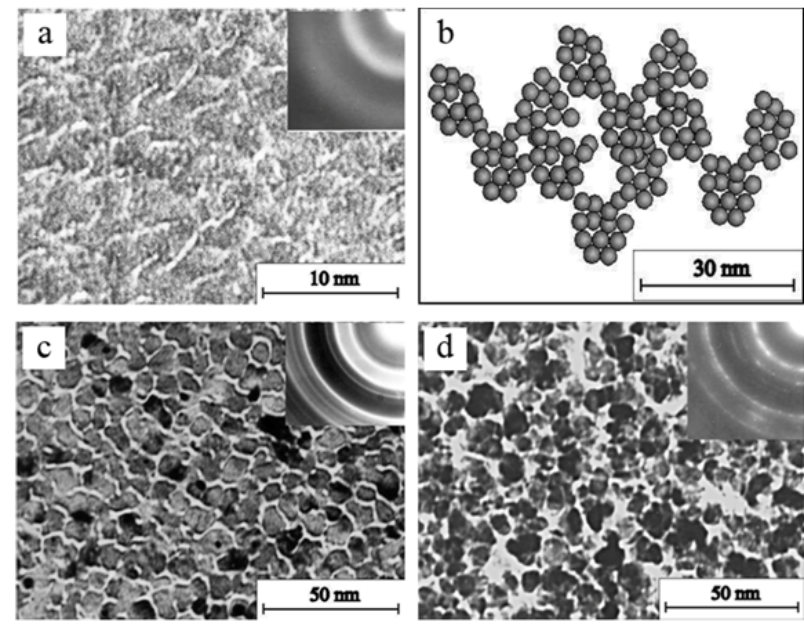

$$
\mathrm{a}, \mathrm{b}-\mathrm{T}_{\text {sub }}=100{ }^{\circ} \mathrm{C} ; \mathrm{c}-\mathrm{T}_{\text {sub }}=300^{\circ} \mathrm{C} ; \mathrm{d}-\mathrm{T}_{\text {sub }}=400{ }^{\circ} \mathrm{C}
$$

Fig. 1. Effect of the substrate temperature $T_{s u b}$ on the tin dioxide film structure shown by electron microscopy images and electron diffraction patterns (inserts).
Electron diffractions of the samples obtained at $T_{\text {sub }}=300{ }^{\circ} \mathrm{C}$ detected continuous Debye rings and blurred diffuse halo (Fig. 1c, insert), indicating the simultaneous presence of finely crystalline and amorphous components of the $\mathrm{SnO}_{2}$ condensate in these samples.

Sometimes, within a single flat particle the elements with sizes much smaller than the characteristic dimensions of the flat particles are visible contrastingly. This phenomenon can be explained by a block structure of the flat particles. The abovementioned diffraction contrast is caused by the electron diffraction in the individual crystalline blocks of the flat particles. In this case, unlike the little heated conglomerates consisting of amorphous globules isolated one from another by layers of the adsorbed atomizing gases, the flat particles are composed of disoriented but packed crystalline blocks having the same size as that of amorphous globules in the little heated films.

Part of the blocks apparently was unable to crystallize, which explains the presence of amorphous phase in these samples. That is, in this case we can see selective crystallization inside the globules, which does not lead to formation of monolithic crystallites throughout the volume of the original conglomerate. Increasing the temperature of the substrate apparently leads, on the one hand, to enhanced surface migration of emerging particles and to their liquid-like coalescence. On the other hand, the temperature growth reduces adsorption of the residual $\mathrm{Ar}$ and $\mathrm{O}_{2}$ gases on the surface of amorphous globules that stimulates their fusion with formation of the block particles. All this leads to a substantial reduction in the surface free energy of the particles as compared with the little heated conglomerates of globules and to crystallization of the blocks, which results in formation of flat particles.

Raising the substrate temperature up to $400{ }^{\circ} \mathrm{C}$ leads to formation of crystalline tin dioxide layers (Fig. 1d). In this case, the obtained $\mathrm{SnO}_{2}$ crystallites have a three-dimensional shape and well-defined edges as compared with the particles in Fig. 1c, and their dimensions in the film plane are similar to the size of the conglomerates in the little heated films. Diffraction contrast image shows (Fig. 1d, insert) single block crystalline grains; there is an absence of individual units within the majority of the crystallites. Therefore, the coherent scattering regions (approximately $15 \mathrm{~nm}$ ) in such films coincide well with sizes of the isolated particles. Electron diffraction indicates the absence of the amorphous phase in these films. Inhomogeneous distribution of intensity along the rings in the electron diffraction images indicates appearance of texture in the $\mathrm{SnO}_{2}$ condensate, which can be attributed to the beginning of intensive coalescence.

As it was discovered during the magnetron sputtering of the gas sensitive $\mathrm{SnO}_{2}$ films on the pyroceramic substrate with continuous control of their conductivity $G$, the phenomenon of a sharp increase in $G$ (by several times, the so-called $\Delta G$ jump) when $\mathrm{SnO}_{2}$ 
layer reaches a certain critical film thickness $h_{c r}$ was observed. Electron microscopy, electron diffraction and $\mathrm{X}$-ray diffraction studies of the films with different thicknesses grown on the $\mathrm{NaCl}$ single crystal and separated from the substrate have shown that $\mathrm{SnO}_{2}$ films with thicknesses $h<h_{c r}$ are amorphous, while if the film thicknesses $h>h_{c r}$, then $\mathrm{SnO}_{2}$ layer contained both amorphous and crystalline phases (Fig. 2).

These data suggest that the size effect in crystallization is manifested [14], which consists of the fact that when the thickness of the amorphous films exceeds $h_{c r}$ a phase relaxation occurs, which expressed in their partial crystallization. Feature of the phase relaxation in these films is its size-selectivity, namely, preservation of the amorphous state in a certain part of the globules.

We explain this effect of selective stabilization of nonequilibrium phase by the impurity-dimensional factor [14]: the concentration of impurity gases adsorbed on $\mathrm{SnO}_{2}$ surface and their influence increase with decreasing of $\mathrm{SnO}_{2}$ particle size. Experimentally, we have found that the oxygen gas concentration $C_{\mathrm{O}_{2}}$ in

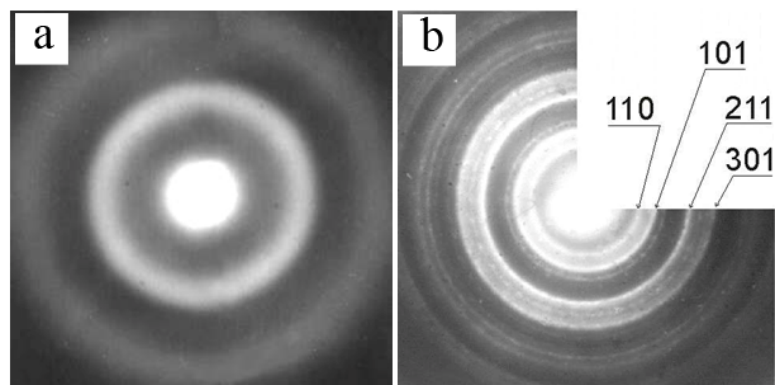

Fig. 2. Electron diffraction patterns for tin dioxide samples with different $\mathrm{SnO}_{2}$ film thicknesses: $h<h_{c r}(a) ; h>h_{c r}(b)$.

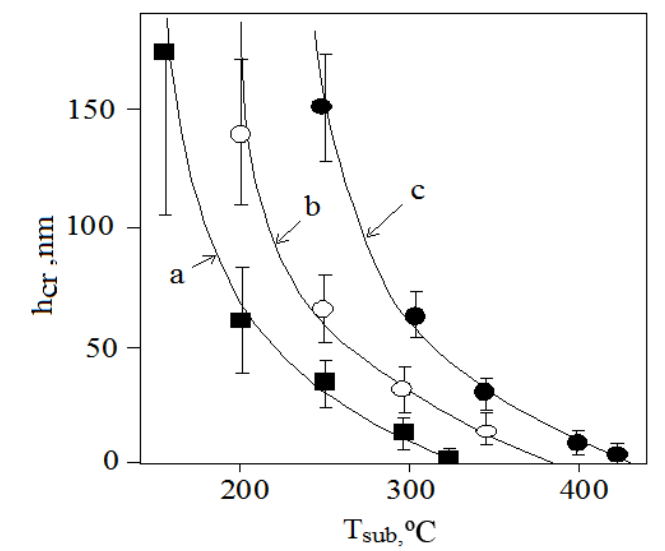

$\mathrm{a}-\mathrm{C}_{\mathrm{O} 2}=20 \% ; \mathrm{b}-\mathrm{C}_{\mathrm{O} 2}=50 \% ; \mathrm{c}-\mathrm{C}_{\mathrm{O} 2}=100 \%$

Fig. 3. Dependence of the critical $\mathrm{SnO}_{2}$ film thickness $h_{c r}$ on the substrate temperature $T_{s u b}$ for the different oxygen concentrations $C_{\mathrm{O}_{2}}$ in the dry $\mathrm{Ar}-\mathrm{O}_{2}$ gas mixture (total gas pressure $2 \mathrm{~Pa}$ ). the Ar- $\mathrm{O}_{2}$ sputtering gas has a significant impact on $h_{c r}$. The dependences $h_{c r}\left(T_{s u b}\right)$ for different values of $C_{\mathrm{O}_{2}}$ are shown in Fig. 3. Furthermore, the presence of water vapor in the $\mathrm{Ar}-\mathrm{O}_{2}$ gas mixture with $C_{\mathrm{O}_{2}}=50 \%$, which fed into the magnetron sputtering operation volume results in the $h_{c r}$ increase up to $100 \ldots 150 \mathrm{~nm}$ for the films grown at $T_{s u b}=250{ }^{\circ} \mathrm{C}$, as compared with $h_{c r} \approx$ $70 \mathrm{~nm}$ when the $\mathrm{Ar}-\mathrm{O}_{2}$ gas mixture was subjected to the conventional purification from water vapor impurities by means of silica gel filter.

Electron microscopic studies of replicas obtained from tin dioxide films with the thickness $h_{c r}$, when the condensation process was stopped immediately after the jump in electric conduction of the growing $\mathrm{SnO}_{2}$ film were fulfilled. They showed (Fig. 4a) an occurrence of "openwork" structure characteristic elements on the surface of these films, which are typical for an explosive crystallization [14]. A high rate of the electrical conductivity change ( $\Delta G$ jump occurs in less than $0.01 \mathrm{~s}$ ) when the film thickness equal to $h_{c r}$ also confirms this phenomenon. Comparing morphology of these surface "openwork" patterns (Fig. 4a) with morphology of the completely crystallized $\mathrm{SnO}_{2}$ film (Fig. 4b), we interpret these patterns as chains of the crystallized particles with the size $L=20 \ldots 30 \mathrm{~nm}$ of each particle, which causes the change in the electrical properties of thin films. The observed phenomenon of explosive partial crystallization is initiated by a size factor and includes an element of randomness, so it may explain a partial irreproducibility of the electrical properties of tin dioxide films taking place even at a very strict control of the parameters during their magnetron sputtering.

The X-ray analysis of the $\mathrm{SnO}_{2}$ films with a thickness by several times exceeding $h_{c r}$ indicates a simultaneous presence of amorphous and crystalline phases. The crystalline phase is in a highly dispersed state (all tested films have averaged coherent scattering regions less than $30 \mathrm{~nm}$ ). Comparison of the results of $\mathrm{X}$-ray diffraction and electron microscopic analysis reveals the block structure of crystalline particles in these $\mathrm{SnO}_{2}$ films. The block sizes are determined by the magnetron sputtering parameters. With the increase in $T_{\text {sub }}$ during $\mathrm{SnO}_{2}$ condensation, the sizes of the coherent scattering regions increase and the content of the amorphous phase in the samples decreases. Thus, the substrate temperature stimulation can explain observed crystallization and recrystallization processes during film growth. The humidity of the atomizing gases, on the contrary, leads to formation of tin dioxide condensate with smaller coherent scattering regions and with increased content of the amorphous phase, indicating this factor as a hindrance for the crystallization process during $\mathrm{SnO}_{2}$ magnetron sputtering.

By means of XRD analysis, the presence of a certain amount of suboxides $\left(\mathrm{SnO}, \mathrm{Sn}_{2} \mathrm{O}_{3}, \mathrm{Sn}_{3} \mathrm{O}_{4}\right)$ was detected on the background of the main tin dioxide phase in the samples with $h>h_{c r}$. As it turned out, the 450 -nm thick films that contained the great amount of 


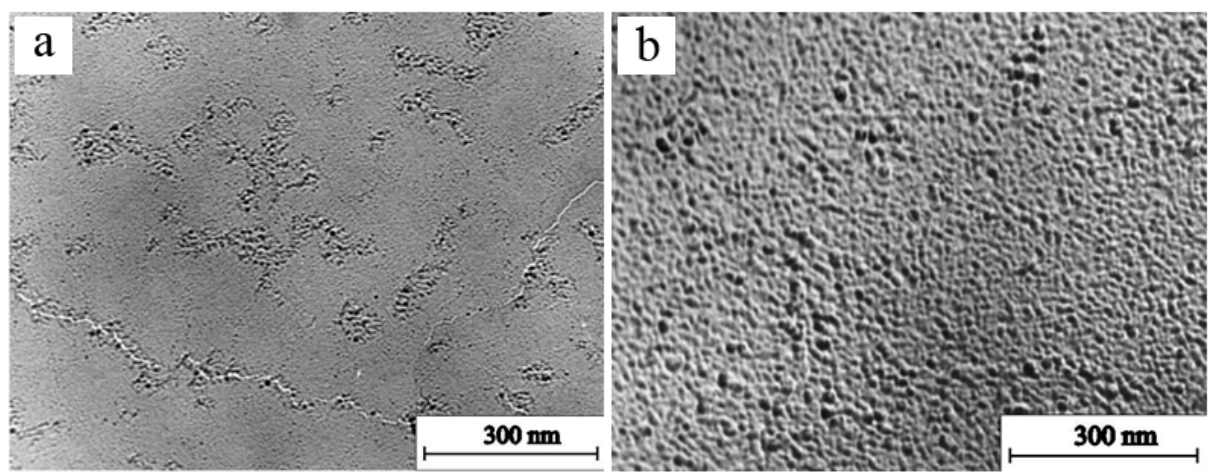

Fig. 4. Electron microscopic image of carbon replicas obtained on $\mathrm{SnO}_{2}$ films with thicknesses $h \approx h_{c r}(a)$ and $h>>h_{c r}(b)$.

suboxides $C_{x}$ were obtained at $T_{s u b}=200{ }^{\circ} \mathrm{C}$ or at $T_{\text {sub }}=$ $250{ }^{\circ} \mathrm{C}$ depending on the concentration of oxygen in the gas mixture (Fig. 5). The amount of suboxides in the magnetron sputtered $\mathrm{SnO}_{2}$ films decreases with increasing the oxygen gas concentration in the atomizing $\mathrm{Ar}-\mathrm{O}_{2}$ gas mixture.

Since in accordance with the model of inter-grain potential barrier $[6,7]$, the gas sensing mechanism of metal oxide semiconductor gas sensors depends on the surface reaction between chemisorbed gases, so the prepared $\mathrm{SnO}_{2}$ films necessarily need to be stable and must have a surface with a large number of adsorption centers, be nanocrystalline and single phase to the extent possible. In the case of direct current magnetron sputtering of $\mathrm{SnO}_{2}$ target in $\mathrm{Ar}-\mathrm{O}_{2}$ gas atmosphere, the above requirements are met by the following conditions: substrate temperature $T_{s u b}=400{ }^{\circ} \mathrm{C}, \quad$ oxygen concentration $C_{\mathrm{O}_{2}}$ higher than $50 \%$, tin dioxide thickness exceeds by several times the value $h_{c r}$, at which abrupt increasing the film conductivity ( $\Delta G$ jump) occurs.

\subsubsection{Effect of annealing on the structure and electric conductivity of the tin dioxide films}

The X-ray diffractometry study of the structure and phase composition of the tin dioxide samples before and after annealing showed that the sample series $\mathrm{S} 1 \quad\left(T_{\text {sub }}=\right.$ $150{ }^{\circ} \mathrm{C}$ ) consisted entirely of amorphous phase before annealing. The amounts of amorphous and crystalline phases in the sample series S2 $\left(T_{\text {sub }}=250^{\circ} \mathrm{C}\right)$ were $85 \%$ and $15 \%$, respectively, and the quantities of the tetragonal crystal phase $\mathrm{SnO}_{2}$ and suboxides $\left(\mathrm{SnO}, \mathrm{Sn}_{2} \mathrm{O}_{3}, \mathrm{Sn}_{3} \mathrm{O}_{4}\right)$ were roughly equal. Tin dioxide samples that were obtained at $T_{\text {sub }}=350^{\circ} \mathrm{C}$ (S3 sample series) consisted almost entirely of the $\mathrm{SnO}_{2}$ crystalline tetragonal phase (content of suboxides was not higher than 3\%). It was found that the air annealing at $T_{\text {sub }}=500{ }^{\circ} \mathrm{C}$ for 1 hour resulted in reducing the amorphous phase content in the samples of S1 and S2 series by about $20 \%$. It should also be noted that the suboxides quantity in the samples of S2 and S3 series decreased by half after annealing at $T_{\text {sub }}=$
$500{ }^{\circ} \mathrm{C}$. It was shown using the electron microscopy that the "witnesses" of the sample of $\mathrm{S} 4$ series grown on $\mathrm{NaCl}$ were amorphous. When heated by an electron beam directly in the electron microscope column, they fully crystallized with a crystallite sizes of $2 \ldots 15 \mathrm{~nm}$. In this case, formation of $\mathrm{SnO}_{2}$ crystal phase was accompanied by the presence of certain amount of suboxides. This is evidenced by the presence of weak intensity diffraction lines (less than $10 \%$ of line of the maximum intensity) in the electron diffraction pattern which are characteristic for the suboxides but not for $\mathrm{SnO}_{2}$. When interpreting the results of structural investigations of S1, S2 and S4 film samples, it should be taken into account that the fine crystalline phase with crystallite sizes of 2 to $15 \mathrm{~nm}$ is recorded as X-ray amorphous. Therefore, we suppose that the presence of the significant amount of amorphous phase in the $\mathrm{S} 1$ and $\mathrm{S} 2$ samples after annealing in air at $500{ }^{\circ} \mathrm{C}$ indicates formation of the fine-grained $\mathrm{SnO}_{2}$ phase.

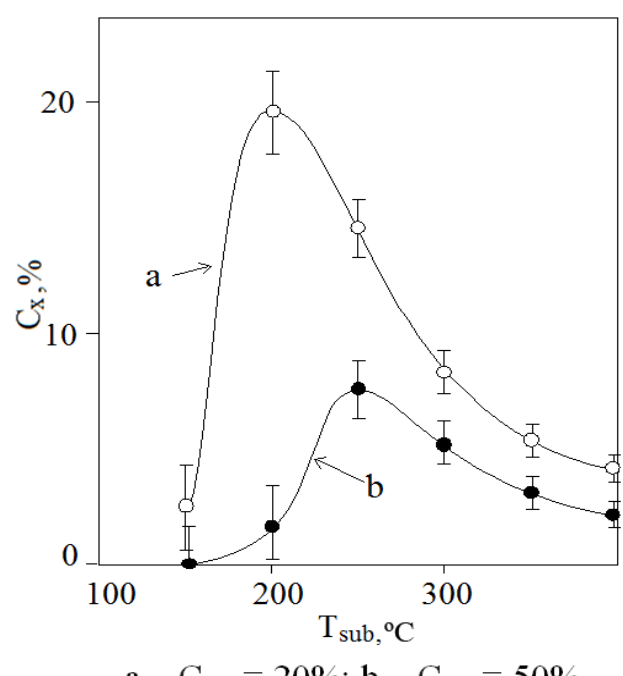

Fig. 5. Influence of the substrate temperature $T_{s u b}$ on the relative amount of suboxides $C_{x}$ in the tin dioxide films obtained by magnetron sputtering at various oxygen gas concentrations in the atomizing $\mathrm{Ar}-\mathrm{O}_{2}$ gas mixture (total gas pressure $2 \mathrm{~Pa}$ ). 
It was found that post-condensation annealing in air resulted in a change of conductivity of tin dioxide films from $G_{0}$ to $G$ due to a change in their crystal structure. Change of the electric conductivity is irreversible and may also be associated with experimentally recorded crystallization of the amorphous phase and with the change in tin dioxide and tin suboxides phase ratio after annealing. It should be noted that formation of the fine crystalline phase with the developed grain-boundary surface can intensify adsorption and desorption processes of atoms and molecules from the environment. The conductivity change during annealing caused by the growing concentration of charge carriers inside the microcrystals and adsorption-desorption processes on the surface is reversible. So, the observed change of the tin dioxide sample conductivity should be repeated upon subsequent heating. Comparison of the temperature dependences of the electric conductivity obtained upon first and subsequent annealing allowed to separate both abovementioned physical processes. To this end, the conductivity measurements of S1, S2, S3 and S4 samples were performed during annealing (Fig. 6a). Analysis of the experimental results showed that all curves are characterized by maximums regardless of the sample deposition temperature. Repeated measurement of electrical conductivity of the films during the reannealing (Fig. 6b) showed that the behavior of $G / G_{0}$ remained unchanged indicating that the reversible absorption-desorption physical mechanism was implemented. In our opinion, the extreme dependence of $G / G_{0}$ is caused by the following reversible competing physical processes at higher temperature. Thermostimulated ionization of the surface states (mainly chemisorbed hydroxyl groups in concerned temperature interval) can lead to an increase in the charge carrier concentration and decrease in the grain potential barrier height [15]. These processes lead to formation of the experimentally observed area of the monotonically increased conductivity. On the other side, the temperature increase over $450{ }^{\circ} \mathrm{C}$ stimulate desorption of the hydroxyl groups, which leads to formation of the area with $G / G_{0}$ monotonic decreasing. It should be noted that the intensity of the above described processes grows with the increase of the grain-boundary surface.

It was shown experimentally, that during the first annealing the value of the conductivity gain maximum $G / G_{0}$ decreased, and the maximum position shifted towards the higher temperatures $T_{\text {sub }}$ for the tin dioxide films with equal thicknesses $h$. The dependence of the conductivity change on the film deposition temperature is due to the fact that the deposition temperature determines the content of the tin suboxides as well as the ratio between the amorphous and crystalline tin dioxide phases in the films. When re-annealing all the $G / G_{0}$ maxima correspond to the same temperature $275^{\circ} \mathrm{C}$. Structural studies of the films showed an increase in the content of the $\mathrm{SnO}_{2}$ crystalline phase in the initial state in the samples with $T_{\text {sub }}$ growing.

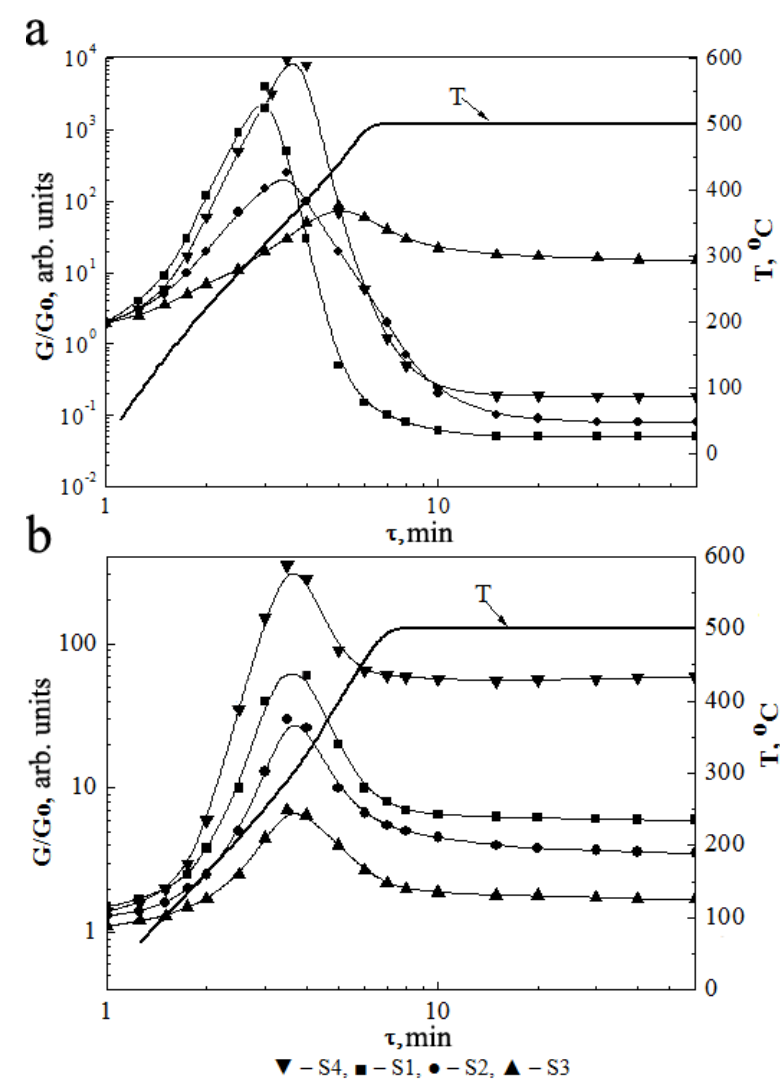

Fig. 6. Time dependences of the annealing temperature $T$ and relative changes of the electric conductivities $G / G_{0}$ for the samples of S1-S4 series upon the first $(a)$ and subsequent annealing $(b)$ in air.

We have noted that the crystallization of the amorphous films of S4 series $(h=50 \mathrm{~nm})$ starts at higher temperatures than that in the more thick $\mathrm{SnO}_{2}$ films, namely for the samples of S1 series $(h=450 \mathrm{~nm})$ deposited at the same temperature $T_{s u b}=150{ }^{\circ} \mathrm{C}$. This is a manifestation of stabilization of the thermodynamically nonequilibrium structure in thin films due to the size factor. In addition, the value of the $G / G_{0}$ maximum decreases with $T_{s}$ due to the grainboundary surface reduction upon the crystallization of the amorphous phase. This conclusion was supported by studies of amorphous 50-nm thick films of S4 series, which have the highest value of the maximum among all the investigated samples (see Figs $6 a$ and $6 b$ ).

Therefore, the tin dioxide active layer for the resistive gas sensor can be made by means of formation of the amorphous layer with the 50-nm thickness on the surface of the $450-\mathrm{nm}$ thick $\mathrm{SnO}_{2}$ film and upon subsequent annealing of this structure in air at $T=$ $500{ }^{\circ} \mathrm{C}$ for 1 hour.

\subsection{Zinc oxide nanostructures}

Nanocrystalline and single phase zinc oxide nanostructures with high surface area which are 
promising for the development of resistive gas sensors, as it was shown, can be created without the use of annealing but just by varying the pulsed electrochemical deposition process parameters.

\subsubsection{The influence of the pulse frequency on the structure of $\mathrm{ZnO}$ arrays}

Analysis of the X-ray diffraction patterns of electrodeposited zinc oxide arrays allowed us to conclude that all of the arrays consisted of single hexagonal $\mathrm{ZnO}$ phase modification (JCPDS 36-1451). Studies of the influence of pulse repetition frequency of the cathode potential on the structural and substructural parameters of $\mathrm{ZnO}$ show that the pulse frequency has no profound effect on the crystal lattice parameters, crystallite size and microstrain in the arrays of zinc oxide (Fig. 7, Table). Small regions of coherent scattering manifest themselves in broadening of the diffraction peaks in Fig. 7. Calculations show that all arrays of the electrodeposited $\mathrm{ZnO}$ layers are nanocrystalline (the corresponding CSRs do not exceed tens or hundreds of nanometers) and exhibit slightly larger crystal lattice parameters compared to those of the reference $\mathrm{ZnO}$ material (according to JCPDS 36-1451, $a=3.250 \AA$ and $c=5.207 \AA)$ and insignificant compression microstrains (Table). The increase in the pulse frequencies $f$ above $2 \mathrm{~Hz}$ decreased the $\langle 001\rangle$ preferred orientation of the $\mathrm{ZnO}$ arrays. The vertical preferential orientation of $\mathrm{ZnO}$ nanocrystals with $c$ axis perpendicular to the substrate $(\langle 001\rangle$ texture $)$ is pronounced in the diffraction patterns (Fig. 7) as intense peaks corresponding to the planes (002) and (004). Note that in [16] we have previously demonstrated a reduction in $\langle 001\rangle$ texture perfection at frequencies below $2 \mathrm{~Hz}$. So, onwards we consider in more detail the $\mathrm{ZnO}$ films electrodeposited with cathode pulse frequencies $f=$ $2 \ldots 20 \mathrm{~Hz}$.

\subsubsection{The influence of the electrolyte temperature and duty cycle on the ZnO surface morphology}

Increasing the temperature of electrolyte $t$ within the range from 65 to $85^{\circ} \mathrm{C}$ resulted in the growth of well textured in the $\langle 001\rangle$ zinc oxide arrays (Table). While the sample grown at $t=60^{\circ} \mathrm{C}$ was very poorly textured in $\langle 102\rangle$ and $\langle 103\rangle$ directions, the arrays of zinc oxide electrodeposited at $t=70{ }^{\circ} \mathrm{C}$ and $85^{\circ} \mathrm{C}$ shown in Figs 8a
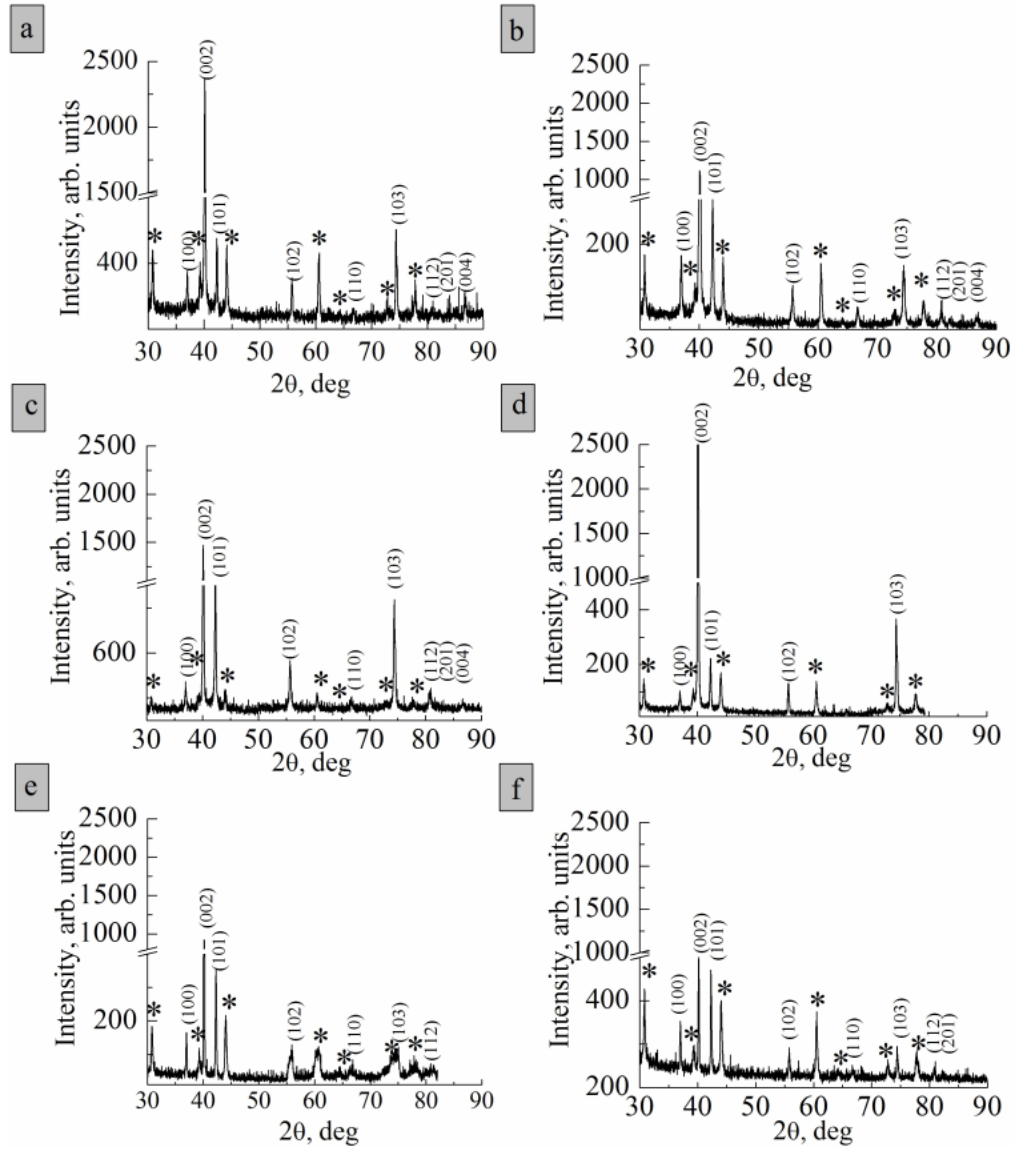

Fig. 7. X-ray diffraction patterns of $\mathrm{ZnO}$ arrays electrodeposited onto glass substrates coated with transparent conductive oxide (asterisks * indicates FTO) at $t=70{ }^{\circ} \mathrm{C}, D c=40$ and different frequencies $f: 2 \mathrm{~Hz}(a) ; 6 \mathrm{~Hz}(b) ; 10 \mathrm{~Hz}(c) ; 20 \mathrm{~Hz}(d)$; $200 \mathrm{~Hz}(e) ; 1 \mathrm{kHz}(f)$. 
Table. Electrodeposition parameters and structure characteristics of pulse plated $\mathrm{ZnO}$ arrays.

\begin{tabular}{|c|c|c|c|c|c|c|c|}
\hline \multicolumn{3}{|c|}{ Plating parameters } & \multicolumn{5}{c|}{ Structure of ZnO arrays } \\
\hline \multirow{2}{*}{$t,{ }^{\circ} \mathrm{C}$} & $f, \mathrm{~Hz}$ & $D c$ & $P_{(002)}$ & \multirow{2}{*}{$C S R, \mathrm{~nm}$} & \multirow{2}{*}{$\Delta d / d, 10^{-3}$} & \multicolumn{2}{c|}{ Lattice parameters, } \\
\cline { 4 - 8 } & & & & & & $a$ & \\
\hline 60 & 1000 & 0.4 & 2.13 & $60-100$ & $0.3-0.6$ & 3.253 & 5.215 \\
\hline 70 & 200 & 0.4 & 2.01 & $19-66$ & $6.4-7.9$ & 3.250 & 5.206 \\
\hline 70 & 20 & 0.4 & 3.12 & $73-163$ & $0.3-0.6$ & 3.253 & 5.220 \\
\hline 70 & 10 & 0.4 & 3.39 & $40-45$ & $0.9-1.4$ & 3.257 & 5.218 \\
\hline 70 & 6 & 0.4 & 4.91 & $73-150$ & $0.1-0.8$ & 3.258 & 5.215 \\
\hline 80 & 5 & 0.4 & 4.88 & $>200$ & - & 3.257 & 5.217 \\
\hline 70 & 3 & 0.4 & 5.25 & $>200$ & - & 3.259 & 5.217 \\
\hline 70 & 2 & 0.4 & 5.43 & $60-80$ & $0.5-0.8$ & 3.256 & 5.215 \\
\hline 50 & 2 & 0.6 & - & $20-30$ & $0.9-1.9$ & 3.258 & 5.211 \\
\hline 30 & 2 & 0.6 & 2.14 & $20-30$ & $1.3-2.4$ & 3.259 & 5.217 \\
\hline 60 & 2 & 0.6 & - & $40-70$ & $0.6-1.1$ & 3.255 & 5.218 \\
\hline 85 & 2 & 0.4 & 4.00 & $60-100$ & $0.2-0.4$ & 3.256 & 5.213 \\
\hline 70 & 2 & 0.2 & 4.00 & $127-200$ & $0.5-1.1$ & 3.258 & 5.215 \\
\hline 70 & 2 & 0.6 & 5.22 & $90-100$ & $0.3-1.1$ & 3.257 & 5.217 \\
\hline 70 & 2 & 0.8 & 3.21 & $40-65$ & $0.3-1.1$ & 3.257 & 5.212 \\
\hline
\end{tabular}

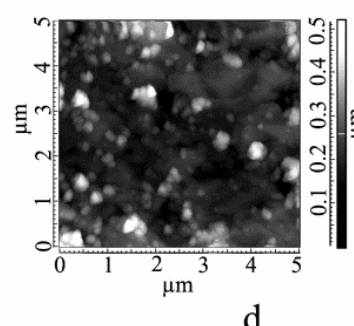

b

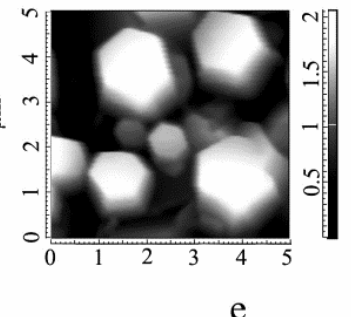

e c

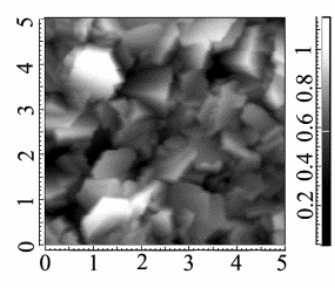

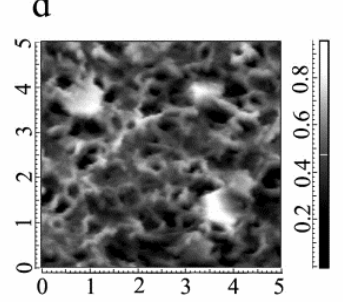

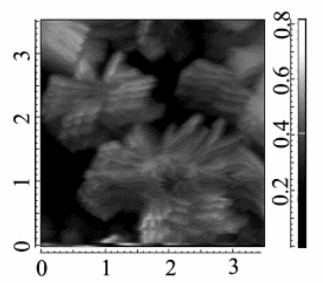

Fig. 8. AFM images of $\mathrm{ZnO}$ arrays from Table deposited at $f=2 \mathrm{~Hz}$ and: (a) $t=70{ }^{\circ} \mathrm{C}, D c=0.4$; (b) $t=85^{\circ} \mathrm{C}, D c=0.4$; (c) $t=$ $70{ }^{\circ} \mathrm{C}, D c=0.8$; (e) $t=77 \rightarrow 70{ }^{\circ} \mathrm{C}, D c=0.4$.

and $8 \mathrm{~b}$, respectively, consisted of nanorods oriented perpendicular to the substrate surface, but they had the perfect texture in $\langle 001\rangle$. As can be seen in Fig. 8, nanorods became larger as the temperature of the electrolyte increased, the diameter of the hexagonal base increased from $100 \mathrm{~nm}\left(t=70^{\circ} \mathrm{C}\right)$ to $1.5 \mu \mathrm{m}\left(t=85^{\circ} \mathrm{C}\right)$.

To vary morphology of the electrodeposited zinc oxide arrays, we used not only the change in temperature of the electrolyte, but also such factor as the duty cycle $D c$ (the ratio of pulse time to its period). It turned out that at the same $f$ and $t$ the preferred orientations, CSRs, and overall morphology of the electrodeposited zinc oxide arrays (Fig. 8) can greatly vary by $D c$ modification. The oriented perpendicular to the substrate zinc oxide nanorods of $100 \mathrm{~nm}$ in diameter have been obtained at $D c=0.4$ (Fig. 7a). The AFM investigations show the stacks of two-dimensional hexagonal nanoplates with diameters of 600 to $1000 \mathrm{~nm}$ and the thickness close to $50 \mathrm{~nm}$, which were formed at $D c=0.6$ (Fig. 8c). If $D c=0.8$, three-dimensional networks of this

\section{(C) 2014, V. Lashkaryov Institute of Semiconductor Physics, National Academy of Sciences of Ukraine}


material with the filament thickness about $100 \mathrm{~nm}$ and with hexagonal network cells of $200 \ldots 600 \mathrm{~nm}$ in diameter have been deposited (Fig. 8d).

Previously [16], we proved that for the preferential growth of zinc oxide arrays of wurtzite modification in the direction perpendicular to the substrate surface, it is necessary to eliminate the adsorption of the hydrogen bubbles formed on the (002) plane during the electrodeposition of $\mathrm{ZnO}$. The results of our studies showed also that in order to ensure desorption of gases from the surface of the growing zinc oxide array to form $\mathrm{ZnO}$ nanorods textured in $\langle 001\rangle$, the electrolyte should be heated to a temperature of at least $70{ }^{\circ} \mathrm{C}$, and, the more heated is electrolyte, the larger 1D nanostructures are formed, due to acceleration and intensification of diffusion and electrochemical processes with temperature. On the other hand, by cooling the electrolyte during electrodeposition from 77 down to $70^{\circ} \mathrm{C}$ the fine zinc oxide nanorods on the surface of the large nanorods were grown and thus hierarchical nanostructures presented in Fig. 8e were created. Another tool to control surface morphology of the electrodeposited zinc oxide arrays for creating the hierarchical nanostructures was duty cycle, because by operating with $T_{o n}$ and $T_{\text {off }}$ ( $T_{o n}$ is a time at the potential $U_{o n} ; T_{\text {off }}$ is that at the potential $\left.U_{\text {off }}\right)$ we can regulate the electrochemical and diffusion stages during $\mathrm{ZnO}$ growth. For example, our studies have shown, in particular, that $\mathrm{ZnO}$ nanorods are formed only if $D c=0.4$, and a structure with a large specific surface in the form of mesoporous networks, as shown in Fig. 8d, is formed at $D c=0.8$. Therefore, by changing the mode of electrodeposition the surface of the $\mathrm{ZnO}$ nanorods can be "encapsulated" by the mesoporous networks of the same material tightly bound with the nanorods both mechanically and electrically, and in this way hierarchical nanostructures are formed with a large surface area for the significant increasing the gas sensitivity.

\section{Conclusions}

Direct current magnetron sputtering of nanoscale tin dioxide films demonstrates that the change in the substrate temperature and in the film thickness leads to the transition from the condensation of metastable conglomerates of amorphous globules to approximately the $15-\mathrm{nm} \quad \mathrm{SnO}_{2}$ crystallites with three-dimensional shape and well-defined edges.

The dependence of the $\mathrm{SnO}_{2}$ structures from the operation $\mathrm{Ar}-\mathrm{O}_{2}$ gas mixtures and from their humidity evidences the significant role of the adsorption in the kinetics of the magnetron sputtering of tin dioxide. Due to adsorption, the morphological and dimensional characteristics of the tin dioxide films demonstrate the specific thermodynamic state, which manifests itself in the anomalous stability of the amorphous globules with their enhanced specific surface energy and stabilization of the amorphous state, selectively retained even after the $\mathrm{SnO}_{2}$ film reaches in general the critical thickness of crystallization.

The quantitative behavior of electrical conductivity depends on the deposition temperature of the films, because it determines the content of tin suboxides, as well as the ratio between the amorphous and crystalline phase of tin dioxide. The $\mathrm{SnO}_{2}$ sample annealing in air at $500{ }^{\circ} \mathrm{C}$ for 1 hour leads to the stabilization of the time dependence of the electric conductivity upon subsequent annealing. The fine crystalline phase with a developed surface is formed in the $\mathrm{SnO}_{2}$ films of the $50-\mathrm{nm}$ thickness $\left(T_{\text {sub }}=150^{\circ} \mathrm{C}\right)$ upon subsequent annealing at $500{ }^{\circ} \mathrm{C}$ due to step-by-step crystallization.

Formation of the amorphous 50-nm thick layer on the surface of the 450-nm thick tin dioxide film and subsequent annealing of this structure in air at $500{ }^{\circ} \mathrm{C}$ for 1 hour allow to create an active layer of the resistive gas sensor with high gas sensitivity, stability and reproducibility.

Another way is the pulsed electrodeposition of zinc oxide as a promising candidate for creation of gas sensors. Studies of effects of pulsed electrodeposition modes on structural and substructural parameters, and on morphology of zinc oxide arrays obtained in aqueous electrolytes made it possible to identify conditions that are optimal to form nanorods of this material, which are oriented perpendicular to the substrate surface. The nanorod sizes can be varied by heating or cooling the electrolyte in the above-mentioned range, making small nanorods electrodeposited on the surface of larger ones and thus forming hierarchical nanostructures. By changing the duty cycle, surface morphology of the arrays is modified up to formation of zinc oxide mesoporous networks, which together with $\mathrm{ZnO}$ nanorods can form hierarchical nanostructures with large specific surface areas and are suitable as active layers of the resistive gas sensors.

\section{References}

1. 1. X. Liu, S. Cheng, H. Liu, S. Hu, D. Zhang and H. Ning, A survey on gas sensing technology // Sensors, 12, p. 9635-9665 (2012).

2. H. Gu, Z. Wang and Y. Hu, Hydrogen gas sensors based on semiconductor oxide nanostructures // Sensors, 12, p. 5517-5550 (2012).

3. J. Gonga, Q. Chena, W. Fei and S. Seal, Micromachined nanocrystalline $\mathrm{SnO}_{2}$ chemical gas sensors for electronic nose // Sensors and Actuators, 102, p. 117-125 (2004).

4. G.E. Patil. D.D. Kajale, D.N. Chavan, N.K. Pawar, P.T. Ahire, S.D. Shinde, V.B. Gaikwad and G.H. Jain, Synthesis, characterization and gas sensing performance of $\mathrm{SnO}_{2}$ thin films prepared by spray pyrolysis // Bull. Mater. Sci., 34(1), p. 1-9 (2011).

5. N. Yamazoe, Toward innovations of gas sensor technology // Sensors and Actuators, 108, p. 2-14 (2005). 
6. A.S. Garde, LPG and $\mathrm{NH}_{3}$ Sensing properties of $\mathrm{SnO}_{2}$ thick film resistors prepared by screen printing technique // Sensors \& Transducers Journal, 122(11), p. 128-142 (2010).

7. N.H. Al-Hardan, M.J. Abdullah, A. Abdul Aziz, Sensing mechanism of hydrogen gas sensor based on RF-sputtered $\mathrm{ZnO}$ thin films // Intern. J. Hydrogen Energy, 35, p. 4428-4434 (2010).

8. A.S. Reddy, N.M. Figueiredo and A. Cavaleiro, Nanocrystalline $\mathrm{Au}: \mathrm{Ag}: \mathrm{SnO}_{2}$ films prepared by pulsed magnetron sputtering // Journal of Physics and Chemistry of Solids, 74, p. 825-829 (2013).

9. M.A. Camacho-López, J.R. Galeana-Camacho, A. Esparza-García, C. Sánchez-Pérez and C.M. Julien, Characterization of nanostructured $\mathrm{SnO}_{2}$ films deposited by reactive DC-magnetron sputtering // Superficies y Vacio, 26(3), p. 95-99 (2013).

10. D. Leng, L. Wu, H. Jiang, Y. Zhao, J. Zhang,W. Li, and L. Feng, Preparation and properties of $\mathrm{SnO}_{2}$ film deposited by magnetron sputtering // Hindawi Publ. Corp. Intern. J. Photoenergy, 2012, Article ID 235971, 6 pages (2012).
11. A.F. Khan, M. Mehmood, A.M. Rana, and M.T. Bhatti, Effect of annealing on electrical resistivity of rf-magnetron sputtered nanostructured $\mathrm{SnO}_{2}$ thin films // Appl. Surf. Sci. 255, p. 85628565 (2009).

12. V.-M. Guérin, J. Rathousky, Th. Rauporté, Electrochemical design of $\mathrm{ZnO}$ hierarchical structures for dye-sensitized solar cells // Solar Energy Materials \& Solar Cells, 102(8), p. 8-14 (2012).

13. J.Y. Lao, J.Y. Huang. D.Z. Wang, Z.F. Ren, Hierarchical oxide nanostructures // J. Mater. Chem. 14, p. 770-773 (2004).

14. L.S. Palatnik, M.J. Fuchs, and V.M. Kosevich, The Mechanism of Formation and Substructure of Condensed Films. Nauka, Moscow, 1972.

15. H. Meixner, U. Lampe, Metal oxide sensors // Sensors and Actuators, 33, p. 198-202 (1996).

16. N.P. Klochko, G.S. Khrypunov, Y.O. Myagchenko, E.E. Melnychuk, V.R. Kopach, E.S. Klepikova, V.M. Lyubov, A.V. Kopach, Controlled growth of one-dimensional zinc oxide nanostructures in the pulsed electrodeposition mode // Semiconductors, 46, p. 825-831 (2012). 